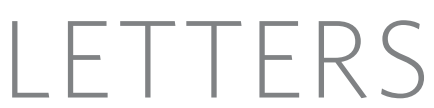

\title{
Drosophila RNAi screen identifies host genes important for influenza virus replication
}

\author{
Linhui Hao ${ }^{1,2 *}$, Akira Sakurai ${ }^{3 * \dagger}$, Tokiko Watanabe ${ }^{3}$, Ericka Sorensen ${ }^{1}$, Chairul A. Nidom ${ }^{5,6}$, Michael A. Newton $^{4}$, \\ Paul Ahlquist ${ }^{1,2} \&$ Yoshihiro Kawaoka ${ }^{3,7,8,9}$
}

All viruses rely on host cell proteins and their associated mechanisms to complete the viral life cycle. Identifying the host molecules that participate in each step of virus replication could provide valuable new targets for antiviral therapy, but this goal may take several decades to achieve with conventional forward genetic screening methods and mammalian cell cultures. Here we describe a novel genome-wide RNA interference (RNAi) screen in Drosophila ${ }^{1}$ that can be used to identify host genes important for influenza virus replication. After modifying influenza virus to allow infection of Drosophila cells and detection of influenza virus gene expression, we tested an RNAi library against 13,071 genes (90\% of the Drosophila genome), identifying over 100 for which suppression in Drosophila cells significantly inhibited or stimulated reporter gene (Renilla luciferase) expression from an influenza-virus-derived vector. The relevance of these findings to influenza virus infection of mammalian cells is illustrated for a subset of the Drosophila genes identified; that is, for three implicated Drosophila genes, the corresponding human homologues ATP6V0D1, COX6A1 and NXF1 are shown to have key functions in the replication of $\mathrm{H} 5 \mathrm{~N} 1$ and $\mathrm{H} 1 \mathrm{~N} 1$ influenza $A$ viruses, but not vesicular stomatitis virus or vaccinia virus, in human HEK 293 cells. Thus, we have demonstrated the feasibility of using genome-wide RNAi screens in Drosophila to identify previously unrecognized host proteins that are required for influenza virus replication. This could accelerate the development of new classes of antiviral drugs for chemoprophylaxis and treatment, which are urgently needed given the obstacles to rapid development of an effective vaccine against pandemic influenza and the probable emergence of strains resistant to available drugs.

Influenza, a highly contagious disease of birds and mammals, is caused by negative-strand RNA viruses of the family Orthomyxoviridae. Influenza outbreaks kill millions of people worldwide during pandemic years and hundreds of thousands during other years. Since their first lethal infection of humans in 1997, H5N1 influenza A viruses have spread throughout Asia and to Europe and Africa, posing a major risk for a new influenza pandemic ${ }^{2}$. To provide rational bases for improved treatment and control of influenza virus infection, we sought to advance understanding of viral infection mechanisms by elucidating previously unknown virus-host cell interactions. Many steps in the viral life cycle, including intracellular trafficking, gene expression, replication and virion assembly, depend on interactions with specific host cell gene products. Although most such host molecules remain elusive, emerging results indicate that their identification and characterization can provide new insights into the mechanisms by which viruses complete their life cycle, and hence illuminate potentially valuable targets for prophylactic and therapeutic intervention ${ }^{3-5}$.

Systematic, genome-wide RNAi analysis offers an exciting tool to identify host genes that function in viral replication. Such analysis is facilitated by well-developed model systems such as Drosophila, the genome of which contains only $\sim 14,000$ genes, nearly all of which can be specifically targeted for high efficiency messenger RNA depletion by double-stranded RNA (dsRNA) libraries ${ }^{1}$. Because of its powerful genetics and conservation with vertebrates, Drosophila has been used to make numerous critical contributions to mammalian cell biology ${ }^{6-9}$. Thus, in principle, Drosophila RNAi studies could accelerate identification of host interactions essential for influenza virus replication.

Because Drosophila D-Mel2 cells do not express the human influenza virus receptor $\alpha 2,6$-linked sialic acid (Supplementary Fig. 1), we predicted that wild-type human influenza virus would not be able to infect them. Indeed, we did not detect viral protein expression by immunofluorescence assays in Drosophila D-Mel2 cells inoculated with influenza virus A/WSN/33 (WSN; H1N1) (data not shown). To bypass this block to wild-type influenza virus entry, we generated a genetically modified virus, Flu-VSV-G-GFP (FVG-G), in MadinDarby canine kidney (MDCK) cells by replacing the receptor-binding haemagglutinin (HA) and neuraminidase (NA) genes with genes encoding vesicular stomatitis virus glycoprotein G (VSV-G) and enhanced green fluorescence protein $(e G F P)$, respectively ${ }^{10,11}$ (Fig. 1a). Because the envelopes of the resulting virions bear VSV-G, which mediates entry into mammalian, Drosophila and other cells ${ }^{12}$, FVG-G virions should readily infect Drosophila cells. Twentyfour hours after infection, GFP fluorescence was detected in FVG-Ginfected Drosophila D-Mel2 cells (Supplementary Fig. 2). We also confirmed by real-time polymerase chain reaction (PCR) that influenza virus RNA replication occurred in Drosophila cells (Supplementary Fig. 3). However, Drosophila cells infected with FVG-G did not release detectable virions into the medium, as assayed by infectivity tests on MDCK cells and by electron microscopy (data not shown). This was due, at least in part, to failure of Drosophila cells to express some viral proteins required for virion assembly and infectivity (Supplementary Fig. 4). Thus, Drosophila cells can support influenza virus replication from post-entry to at least the protein expression phase of the viral life cycle. This span encompasses multiple other steps in the life cycle, including cytoplasmic release of 
genomic RNA-containing viral ribonucleoprotein complexes (vRNPs), vRNP import into the nucleus, mRNA synthesis from the negative-strand viral RNA genome, mRNA export to the cytoplasm and translation.

For high-throughput, functional genomics analysis of influenza virus replication in Drosophila cells, we engineered Flu-VSV-GR.Luc (FVG-R), in which VSV-G and Renilla luciferase genes replaced the viral $H A$ and $N A$ open reading frames (Fig. 1b). FVG$\mathrm{R}$ virions were then used with an RNAi library (Ambion) against 13,071 Drosophila genes ( $~ 90 \%$ of all genes) to identify host genes affecting influenza-virus-directed Renilla luciferase expression (Fig. 1c). Two independent tests of the entire library were performed (Supplementary Table 1). For 176 genes for which dsRNAs inhibited FVG-R-directed luciferase expression in both replicates, repeated secondary tests using alternate dsRNAs to control for possible offtarget effects confirmed the effects of 110 genes (Supplementary Tables 2 and 3). This confirmation rate is comparable to that in a Drosophila screen with a natural Drosophila-infecting virus ${ }^{5}$. Cell viability testing identified six genes with potentially significant

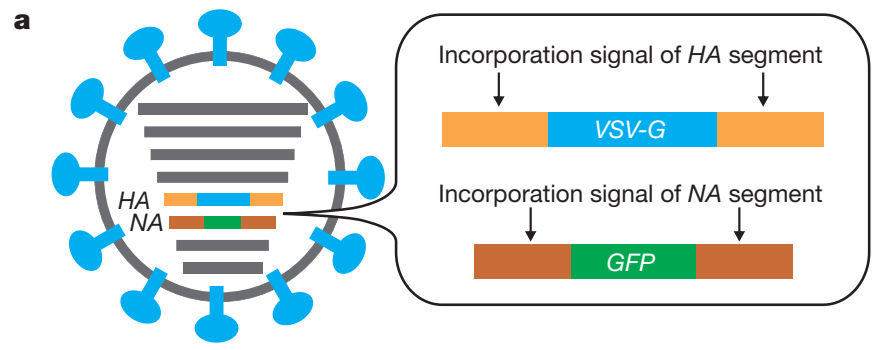

b
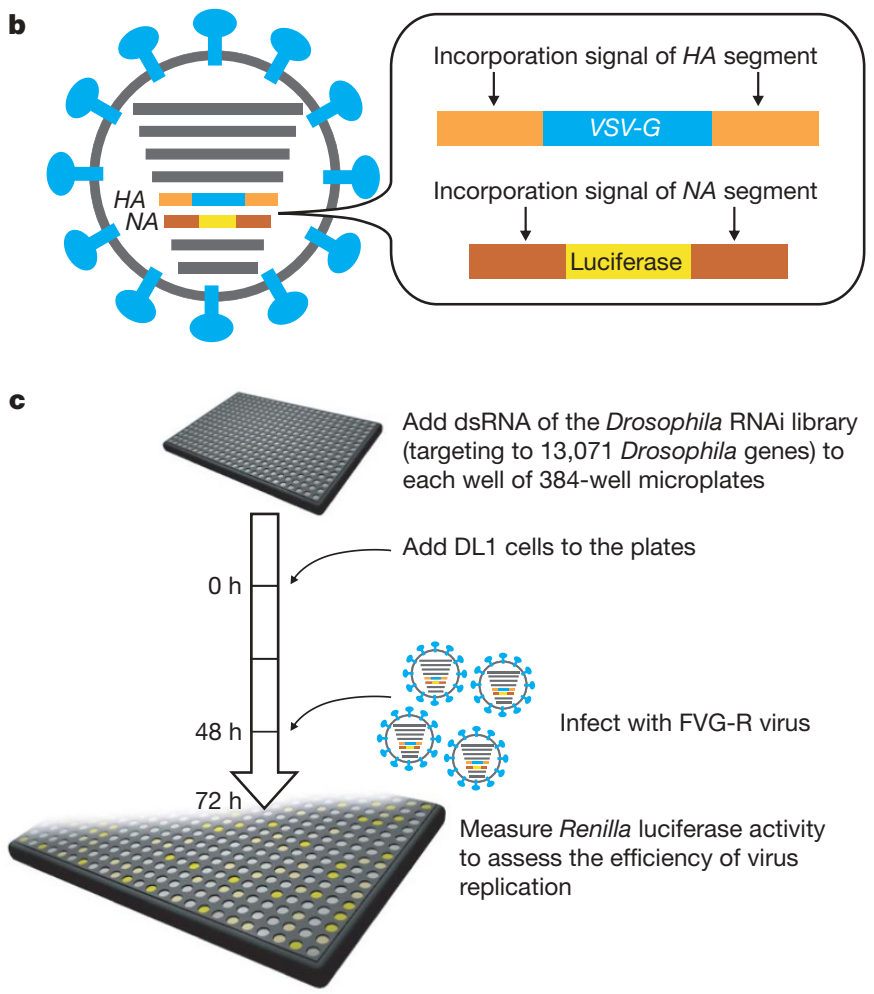

Figure 1 | Overview of genome-wide RNAi screen to identify host factors involved in influenza virus replication in Drosophila cells. a, b, Schematic diagrams showing recombinant influenza viruses. Shown are FVG-G, in which genes encoding the HA and NA proteins were replaced with the VSV$G$ and $e G F P$ genes, respectively (a), and FVG-R, in which the genes encoding the HA and NA were replaced with the $V S V-G$ and Renilla luciferase genes, respectively (b). c, Schematic diagram of the systematic analysis of host genes affecting influenza virus replication and gene expression in Drosophila cells. Experimental details are given in Methods. cytotoxic effects; these were excluded from further consideration (Supplementary Information and Supplementary Table 3). Secondary tests of candidate genes for which dsRNAs increased FVG-R-directed luciferase expression produced a much lower confirmation rate, suggesting a higher rate of off-target or other falsepositive effects in this class (Supplementary Information and Supplementary Table 4).

Among the over 100 candidate genes found to be important for influenza virus replication in Drosophila cells, we selected the human homologues of several encoding components in host pathways/ machineries that are known to be involved in the life cycle of influenza virus, for example, ATP6V0D1 (endocytosis pathway), COX6A1 (mitochondrial function) and NXF1 (mRNA nuclear export machinery), for further analysis in mammalian cells to assess the relevance of our Drosophila results ${ }^{13-17}$. ATP6V0D1 encodes subunit $\mathrm{D}$ of vacuolar $\left(\mathrm{H}^{+}\right)$-ATPase (V-ATPase), a proton pump that functions in the endocytosis pathway (that is, the acidification and fusion of intracellular compartments ${ }^{18}$ ). COX6A1 encodes a subunit of cytochrome $c$ oxidase (COX), an enzyme of the mitochondrial electron transport chain that catalyses electron transfer from cytochrome $c$ to oxygen $^{19}$. NXF1 encodes a nuclear export factor critical for exporting most cellular mRNAs containing exon-exon junctions ${ }^{20,21}$.

As a first test for the possible contribution of these gene products to influenza virus replication in mammalian cells, we treated human HEK 293 cells twice at 24-h intervals with short interfering RNAs (siRNAs; siGENOME, Dharmacon) against the human homologue of each selected Drosophilia gene. Twenty-four hours after the second siRNA treatment, the cells were infected with FVG-R virus and, two days later, Renilla luciferase activity was measured to assess viral replication and gene expression. siRNA against ATP6V0D1 or COX6A1 markedly decreased Renilla luciferase activity (Fig. 2a), but not cell viability (Supplementary Fig. 5a), suggesting that these genes have important roles in influenza virus replication in mammalian cells, as in Drosophila cells. Inhibition was not caused by offtarget effects because, for each gene, each of four distinct siRNAs inhibited FVG-R-directed expression of Renilla luciferase (Supplementary Table 6). Because COX6A1 encodes a subunit of mitochondrial electron transport chain complex IV, COX, we used specific inhibitors to test whether in HEK 293 cells influenza virus also required other complexes in this chain (Fig. 2c). Inhibitors of complexes III, IV and V selectively inhibited FVG-R-directed Renilla luciferase expression by 50 - to 100 -fold, whereas complex I and II inhibitors had little or no effect. Thus, in mammalian cells, influenza virus depends on multiple late stages but not early stages in the mitochondrial electron transport chain.

Treatment for four days with siRNA against NXF1 decreased mammalian cell viability (data not shown), as predicted by the critical role of NXF1 in general host cell metabolism. Accordingly, the total incubation time with siRNA against NXF1 was shortened to $36 \mathrm{~h}$ by transfecting cells with the siRNA twice at a 12-h interval, infecting with FVG-R virus $12 \mathrm{~h}$ later, and assaying for Renilla luciferase at 12-h post-infection. Under these conditions, cell viability was not detectably affected (Supplementary Fig. 5b) whereas Renilla luciferase activity was reduced by nearly fivefold (Fig. 2b). Whereas recent results indicated that influenza virus protein NS1 binds to NXF1 to inhibit host mRNA export ${ }^{17}$, these results imply that influenza virus RNAs and/or proteins are transported by an NXF1-dependent pathway (see also Supplementary Information).

To test the effects of these genes on authentic influenza viruses, we infected siRNA-treated HEK 293 cells with WSN virus or H5N1 influenza A/Indonesia/7/05 (Indonesia 7; isolated from a patient) or with VSV or vaccinia virus as controls. Progeny viruses were collected from the medium at $24 \mathrm{~h}$ (Indonesia $7, \mathrm{VSV}$ or vaccinia virus) or $48 \mathrm{~h}$ (WSN) post-infection and were titrated. Depleting ATP6V0D1 and COX6A1 did not affect VSV or vaccinia virus replication, but decreased the WSN and Indonesia 7 virus yields by $\sim 10$-fold or more (Fig. 3a). Thus, ATP6VOD1 and COX6A1 are required for replication 
of influenza viruses but not VSV and vaccinia virus. Depleting the NXF1 nuclear export factor, again using an accelerated 36-h time line, reduced Indonesia 7 virus titres by 20 -fold (Fig. 3 b), indicating that NXF1 has a critical role in $\mathrm{H} 5 \mathrm{~N} 1$ influenza virus replication. Unfortunately, because WSN grows in HEK 293 cells more slowly than does Indonesia 7 virus, the WSN virus yield at 12 -h post-infection was insufficient to test for an effect of NXF1 depletion (data not shown). In comparison to Indonesia 7 virus, NXF1 depletion had no effect on VSV or vaccinia virus yields (Fig. 3b). Moreover, siRNA against NXF1 inhibited FVG-G, but not adenovirus, which, like influenza virus, depends on nuclear steps for genome transcription and replication (Supplementary Fig. 6). Thus, multiple genes identified in Drosophila cells correspond to important, selective host factors for influenza virus replication in mammalian cells.

The method that we have established, using systematic analysis of a Drosophila RNAi library with confirmation in mammalian cells, can be used to identify host gene products that affect influenza virus replication. This utility was demonstrated in experiments with authentic influenza viruses, including an $\mathrm{H} 5 \mathrm{~N} 1$ virus isolated from a human patient. One of the candidate genes tested in mammalian cells, COX6A1, which encodes a subunit of COX, acts as a critical enzyme in cytochrome- $c$-dependent electron transport in mitochondria. Influenza virus $\mathrm{PB} 2$ polymerase has a mitochondrial targeting signal $^{14}$, and PB1-F2, the second protein encoded by the influenza PB1 gene, was reported to localize to inner and outer mitochondrial membranes and to delay influenza virus clearance by host antiviral responses ${ }^{15}$. Thus, COX6A1 may be involved in PB2- and/or PB1-F2mediated functions in mitochondria. Additionally, influenza vRNP
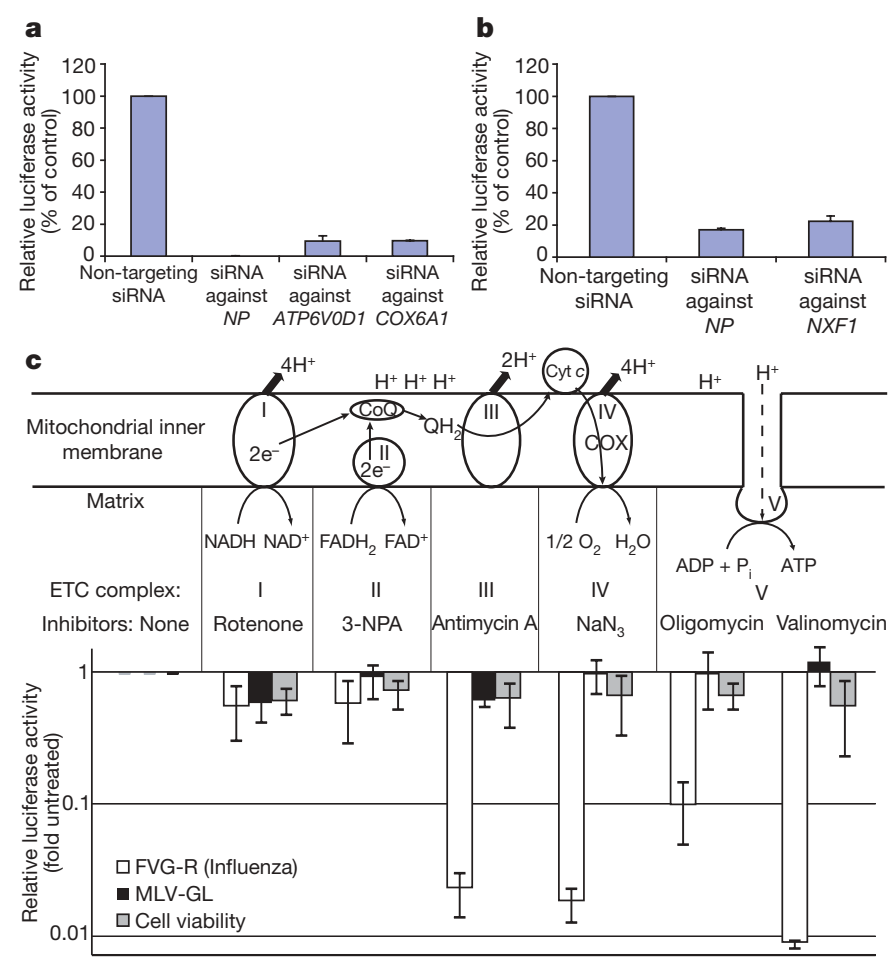

Figure 2 | Effect of selected siRNAs and inhibitors on Renilla luciferase expression in FVG-R-infected human cells. a-c, Renilla luciferase activity was measured in FVG-R-infected HEK 293 cells treated with siRNAs against ATP6V0D1 and COX6A1 (a), NXF1 (b) or the indicated mitochondrial electron transport chain inhibitors (c). Inhibitors of complexes III, IV and V inhibited FVG-R-directed Renilla luciferase expression significantly, whereas complex I and II inhibitors had little or no effect. In contrast, the inhibitors had no significant effects on cell viability and Gaussia luciferase expression of a murine leukaemia virus derivative (MLV-GL) that, like FVG$\mathrm{R}$, depended on VSV G-mediated entry. All experiments were conducted three times in duplicate, with the results reported as mean \pm s.d. 3-NPA, 3-nitroproprionic acid.
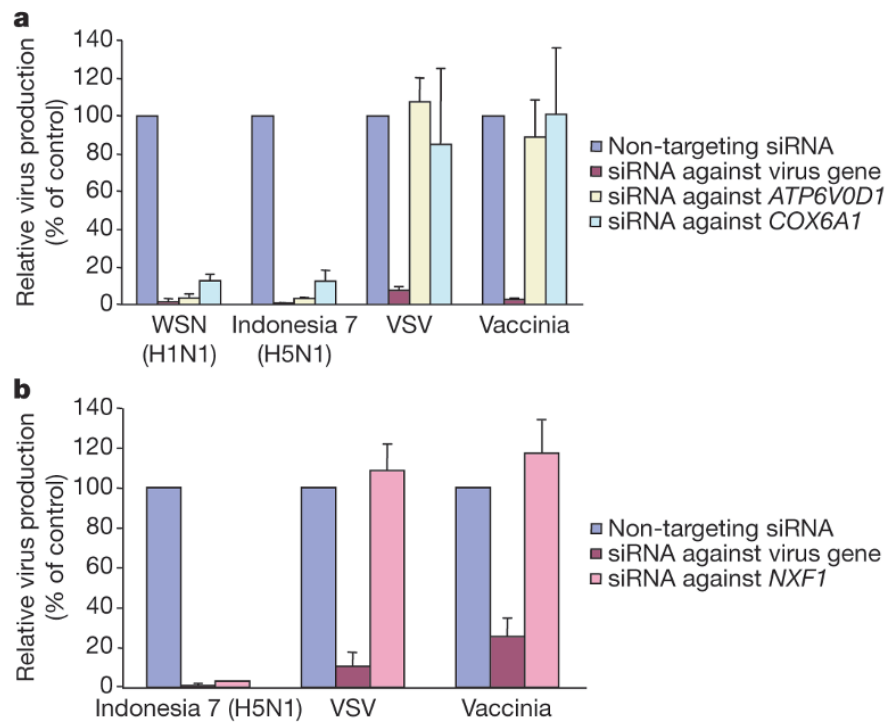

Figure 3 | Effect of siRNAs against selected genes on the replication of influenza viruses, VSV or vaccinia virus in human HEK 293 cells. a, b, The titres of influenza viruses (WSN and Indonesia 7), VSV and vaccinia virus in HEK 293 cells treated with siRNA against ATP6VOD1 and COX6A1 (a) or against NXF1 (b) are shown. Experimental details are given in Methods and Supplementary Methods. All experiments were conducted three times, with the results reported as mean \pm s.d.

export from the nucleus requires caspase 3 activation $^{22}$, which can be induced by cytochrome $c$ release from mitochondria. Moreover, human BCL2 inhibits both cytochrome $c$ release and influenza vRNP export ${ }^{23}$, and COX function and changes in mitochondrial membrane potential have been linked to caspase activation ${ }^{24}$. These pathways may underlie our further findings (Fig. 2c) that influenza virus replication and expression in mammalian cells are strongly and selectively modulated by compounds that inhibit COX, cytochrome $c$-linked electron transport chain complex III, and normal ion transport across mitochondrial membranes (oligomycin and valinomycin). Potential roles of the other two host genes confirmed here in mammalian cells, ATP6V0D1 and NXF1, in influenza virus replication are discussed further in the Supplementary Information.

The above results with multiple, diverse genes, including ATP6V0D1, NXF1 and COX6A1 as well as mitochondrial electron transport complexes III and V, demonstrate the feasibility and value of using Drosophila RNAi screening to identify previously unknown host factors with important and potentially unsuspected roles in influenza virus replication. Simultaneously, the genome-wide results from our Drosophila RNAi screen provide more than 100 additional candidate genes (many with unknown functions) to be tested in mammalian cells. We suggest that the same strategy could be applied to identify previously unknown host factors involved in the replication of other viruses, whenever at least a portion of their replication cycle is supported by Drosophila cells.

\section{METHODS SUMMARY}

Cells and viruses. MDCK cells, HEK 293 cells, baby hamster kidney (BHK) cells, D-Mel2 cells and DL1 cells were maintained as described in Methods. WSN, FVG-G, FVG-R and Indonesia 7 viruses were generated as described previously ${ }^{10}$ and propagated in MDCK cells. VSV and vaccinia virus were grown in BHK and CV-1 cells, respectively. Gaussia-luciferase-expressing murine leukaemia virus (MLV-GL) and the GFP-expressing adenovirus were provided by J. Bruce and R. Kalejta $^{25}$, respectively.

Drosophila RNAi library analysis. Double-stranded RNA of the Drosophila RNAi library (targeting 13,071 Drosophila genes) and DL1 cells were added to each well of 384-well microplates. After two days of incubation, cells were infected with the FVG-R virus. At one day post-infection, Renilla luciferase activity was measured as described below. Two independent analyses of the entire library were performed. 
siRNA treatment of mammalian cells. HEK 293 cells were transfected with siRNA by TransIT-TKO (Mirus). Cells were incubated for $12 \mathrm{~h}$ in experiment with NXF1 or for $24 \mathrm{~h}$ with other genes, were transfected again under the same conditions, and inoculated with virus after $12 \mathrm{~h}$ for NXF1 or after $24 \mathrm{~h}$ for other genes. After a further $12 \mathrm{~h}$ (for NXF1) or $48 \mathrm{~h}$ (other genes), the cells were harvested for the indicated analyses.

Renilla luciferase and cell viability assays. Renilla luciferase activity and cell viability were measured with established Renilla luciferase and CellTiter-Glo assay systems (Promega) according to the manufacturer's instructions; signals were read with a GLOMAX 96 microplate luminometer.

Full Methods and any associated references are available in the online version of the paper at www.nature.com/nature.

\section{Received 15 October 2007; accepted 5 June 2008.}

Published online 9 July 2008.

1. Kuttenkeuler, D. \& Boutros, M. Genome-wide RNAi as a route to gene function in Drosophila. Brief. Funct. Genomic. Proteomic. 3, 168-176 (2004).

2. Webster, R. G. \& Govorkova, E. A. H5N1 influenza - continuing evolution and spread. N. Engl. J. Med. 355, 2174-2177 (2006).

3. Kushner, D. B. et al. Systematic, genome-wide identification of host genes affecting replication of a positive-strand RNA virus. Proc. Natl Acad. Sci. USA 100, 15764-15769 (2003).

4. Ludwig, S., Planz, O., Pleschka, S. \& Wolff, T. Influenza-virus-induced signaling cascades: targets for antiviral therapy? Trends Mol. Med. 9, 46-52 (2003).

5. Cherry, S. et al. Genome-wide RNAi screen reveals a specific sensitivity of IREScontaining RNA viruses to host translation inhibition. Genes Dev. 19, 445-452 (2005).

6. Hahn, H., Wojnowski, L., Miller, G. \& Zimmer, A. The patched signaling pathway in tumorigenesis and development: lessons from animal models. J. Mol. Med. 77, 459-468 (1999).

7. Dearolf, C. R. JAKs and STATs in invertebrate model organisms. Cell. Mol. Life Sci. 55, 1578-1584 (1999).

8. Lemaitre, B., Nicolas, E., Michaut, L., Reichhart, J. M. \& Hoffmann, J. A. The dorsoventral regulatory gene cassette spatzle/Toll/cactus controls the potent antifungal response in Drosophila adults. Cell 86, 973-983 (1996).

9. Takeda, K., Kaisho, T. \& Akira, S. Toll-like receptors. Annu. Rev. Immunol. 21, 335-376 (2003).

10. Neumann, G. et al. Generation of influenza A viruses entirely from cloned cDNAs. Proc. Natl Acad. Sci. USA 96, 9345-9350 (1999).

11. Watanabe, T., Watanabe, S., Noda, T., Fujii, Y. \& Kawaoka, Y. Exploitation of nucleic acid packaging signals to generate a novel influenza virus-based vector stably expressing two foreign genes. J. Virol. 77, 10575-10583 (2003).

12. Wyers, F., Richard-Molard, C., Blondel, D. \& Dezelee, S. Vesicular stomatitis virus growth in Drosophila melanogaster cells: G protein deficiency. J. Virol. 33, 411-422 (1980).

13. Perez, L. \& Carrasco, L. Involvement of the vacuolar $\mathrm{H}(+)$-ATPase in animal virus entry. J. Gen. Virol. 75, 2595-2606 (1994).
14. Carr, S. M., Carnero, E., Garcia-Sastre, A., Brownlee, G. G. \& Fodor, E. Characterization of a mitochondrial-targeting signal in the PB2 protein of influenza viruses. Virology 344, 492-508 (2006).

15. Zamarin, D., Ortigoza, M. B. \& Palese, P. Influenza A virus PB1-F2 protein contributes to viral pathogenesis in mice. J. Virol. 80, 7976-7983 (2006).

16. Momose, F. et al. Cellular splicing factor RAF-2p48/NPI-5/BAT1/UAP56 interacts with the influenza virus nucleoprotein and enhances viral RNA synthesis. J. Virol. 75, 1899-1908 (2001).

17. Satterly, N. et al. Influenza virus targets the mRNA export machinery and the nuclear pore complex. Proc. Natl Acad. Sci. USA 104, 1853-1858 (2007).

18. Stevens, T. H. \& Forgac, M. Structure, function and regulation of the vacuolar (H+)-ATPase. Annu. Rev. Cell Dev. Biol. 13, 779-808 (1997).

19. Gnaiger, E., Lassnig, B., Kuznetsov, A., Rieger, G. \& Margreiter, R. Mitochondrial oxygen affinity, respiratory flux control and excess capacity of cytochrome $c$ oxidase. J. Exp. Biol. 201, 1129-1139 (1998).

20. Cullen, B. R. Nuclear mRNA export: insights from virology. Trends Biochem. Sci. 28, 419-424 (2003).

21. Reed, R. \& Cheng, H. TREX, SR proteins and export of mRNA. Curr. Opin. Cell Biol. $17,269-273$ (2005).

22. Wurzer, W. J. et al. Caspase 3 activation is essential for efficient influenza virus propagation. EMBO J. 22, 2717-2728 (2003).

23. Hinshaw, V. S., Olsen, C. W., Dybdahl-Sissoko, N. \& Evans, D. Apoptosis: a mechanism of cell killing by influenza A and B viruses. J. Virol. 68, 3667-3673 (1994).

24. Kadenbach, B., Arnold, S., Lee, I. \& Huttemann, M. The possible role of cytochrome c oxidase in stress-induced apoptosis and degenerative diseases. Biochim. Biophys. Acta 1655, 400-408 (2004).

25. $\mathrm{He}, \mathrm{T}$. C. et al. A simplified system for generating recombinant adenoviruses. Proc. Natl Acad. Sci. USA 95, 2509-2514 (1998).

Supplementary Information is linked to the online version of the paper at www.nature.com/nature.

Acknowledgements We thank M. McGregor and K. Wells for technical assistance, M. Hatta, S. Yamada and M. Ito for an H5N1 influenza virus, S. Watanabe and Y. Hatta for FVG-R and FVG-G viruses, R. Kalejta and J. Bruce for adenovirus and murine leukaemia virus vectors, T. Noda for electron microscopy, J. Gilbert for editing the manuscript and Y. Kawaoka for illustrations. This work was supported, in part, by US National Institute of Allergy and Infectious Diseases Public Health Service research grants, by the US National Institute of General Medical Sciences grant GM35072, by a grant-in-aid for Specially Promoted Research and by a contract research fund for the Program of Funding Research Centers for Emerging and Reemerging Infectious Diseases from the Ministry of Education, Culture, Sports, Science and Technology, and by grants-in-aid from the Ministry of Health, Labor, Welfare of Japan. P.A. is a Howard Hughes Medical Institute investigator.

Author Contributions L.H., A.S., P.A. and Y.K. designed research; L.H., A.S., T.W. and C.A.N. performed research; L.H., A.S., T.W., E.S., M.A.N., P.A. and Y.K. analysed data; and L.H., A.S., T.W., P.A. and Y.K. wrote the manuscript.

Author Information Reprints and permissions information is available at www.nature.com/reprints. Correspondence and requests for materials should be addressed to Y.K. (kawaokay@svm.vetmed.wisc.edu) or P.A. (ahlqust@wisc.edu). 


\section{METHODS}

Cells and viruses. MDCK cells, HEK 293 cells and BHK cells were maintained in minimum essential medium containing $5 \%$ fetal calf serum and antibiotics at $37^{\circ} \mathrm{C}$ in $5 \% \mathrm{CO}_{2}$. D-Mel2 cells were maintained in Drosophila-SFM (GIBCO/ Invitrogen) at $28^{\circ} \mathrm{C}$. DL1 cells were maintained in Schneider's Drosophila (SD) medium containing $10 \% \mathrm{FBS}$ at $28^{\circ} \mathrm{C}$.

WSN, FVG-G, FVG-R and Indonesia 7 viruses were generated by a plasmidbased reverse genetics system ${ }^{10}$ and were grown and titrated in MDCK cells. MLV-GL was produced by replacing the CD4 open reading frame in pCMMP-CD4-eGFP ${ }^{26}$ with that of Gaussia luciferase (J. Bruce, unpublished observations). All experiments with Indonesia 7 virus were conducted in a biosafety level 3 containment laboratory approved for such use by the Centers for Disease Control and Prevention and the US Department of Agriculture.

Drosophila RNAi library analysis. The Drosophila RNAi library (Ambion) contained 13,071 individual dsRNAs, each designed to specifically target a single Drosophila gene. Five microlitres of dsRNA $\left(40 \mathrm{ng} \mathrm{\mu l}^{-1}\right)$ were added to each well of 384-well plates, after which $2 \times 10^{4}$ DL1 cells in $10 \mu \mathrm{l}$ of SD medium were added to each well and incubated with the dsRNA at $28^{\circ} \mathrm{C}$ for $60 \mathrm{~min}$. Twenty microlitres of SD medium containing $20 \%$ FBS was then added to each well after incubation. Cells were treated with dsRNA for two days at $28^{\circ} \mathrm{C}$ and were then inoculated with an amount of FVG-R virus corresponding to a multiplicity of infection of 10 for MDCK cells, and were then transferred to $33^{\circ} \mathrm{C}$. At one day post-infection, Renilla luciferase activity was measured as described below. Two independent analyses of the entire library were performed.

siRNA treatment of mammalian cells. The non-targeted siRNA used was siCONTROL 1 (Dharmacon). Duplex siRNAs against candidate genes were obtained from siGENOME (Dharmacon, see Supplementary Table 7 for sequences); the effects of siRNA were evaluated by reverse transcription followed by PCR (RT-PCR; Supplementary Fig. 7). The sequences of siRNA against the $N P$ gene of influenza virus (GGAUCUUAUUUCCUUCGGAGUU) ${ }^{27}$ and the E3L gene of vaccinia virus (AAUAUCGUCGGAGCUGUACAC) ${ }^{28}$ were reported previously. The sequence of siRNA against the gene encoding the L protein of VSV (CGAGUUAUCCAGCAAUCAUUU) was designed using BLOCK-iT RNAi designer (Invitrogen). A HEK 293 cell suspension was seeded into the wells of a 24-well plate $\left(2.0 \times 10^{4}\right.$ cells per well), incubated for $1 \mathrm{~h}$ and transfected with siRNA (to a final concentration $10 \mathrm{nM}$; Dharmacon) using TransIT-TKO (Mirus), according to the manufacturer's instructions.

Inhibitor treatment of mammalian cells. Mitochondrial electron transport chain inhibitors were used at the following final concentrations: rotenone $(0.5 \mu \mathrm{M})$, 3-nitroproprionic acid $(1 \mathrm{mM})$, antimycin A $(25 \mu \mathrm{M}), \mathrm{NaN}_{3}$ $(50 \mathrm{mM})$, oligomycin $(25 \mu \mathrm{M})$ and valinomycin $(0.9 \mu \mathrm{M})$. HEK 293 cells were treated with the inhibitors for $5 \mathrm{~h}$ before being infected with FVG-R or MLV-GL, incubated $18 \mathrm{~h}$, and assayed for Renilla or Guassia luciferase, respectively. Cell viability was assayed in parallel by a firefly-luciferase-mediated ATP assay (CellTiter-Glo, Promega).

26. Bruce, J. W., Bradley, K. A., Ahlquist, P. \& Young, J. A. Isolation of cell lines that show novel, murine leukemia virus-specific blocks to early steps of retroviral replication. J. Virol. 79, 12969-12978 (2005).

27. Ge, Q. et al. RNA interference of influenza virus production by directly targeting mRNA for degradation and indirectly inhibiting all viral RNA transcription. Proc. Natl Acad. Sci. USA 100, 2718-2723 (2003).

28. Dave, R. S. et al. siRNA targeting vaccinia virus double-stranded RNA binding protein [E3L] exerts potent antiviral effects. Virology 348, 489-497 (2006). 